\title{
Mucoadhesive in-situ Gel Formulation for Vaginal Delivery of Tenofovir Disoproxil Fumarate
}

\author{
Anita Prahladbhai Patel ${ }^{*}$, Jayvadan K. Patel \\ Department of Pharmaceutics, Faculty of Pharmacy, Nootan Pharmacy College, Sankalchand Patel University, Visnagar, Gujarat, \\ INDIA.
}

\begin{abstract}
Introduction: Tenofovir disoproxil fumarate is an anti-retroviral medicine which belongs to microbicides class being formulated for a woman instigated technique of prevention of the human immunodeficiency virus infection. The objective of the present investigation is to prepare thermosensitive mucoadhesive in-situ vaginal gel of Tenofovir disoproxil fumarate that can present pre-exposure prophylaxis against human immunodeficiency virus in addition to providing excellent spreading as well as coating of the vagina, forming the therapy more effectual and bring about extended effect. Materials and Methods: The vaginal in-situ gel of Tenofovir disoproxil fumarate was prepared using thermosensitive polymer poloxamer 407 and mucoadhesive polymer carbopol 934 by a cold method. It was characterized for drug-excipient compatibility, viscosity, gelation study, gelling capacity, in-vitro drug release study, stability study and Hen's Egg Test-Chorioallantoic Membrane assay. Results and Discussion: Drug excipient compatibility study displayed that there is no interaction between drug and excipients. Formulation F2 was found as the most appropriate formulation on the basis of the evaluation parameters, as it displayed the preferred properties. The work of adhesion values was used as parameters for comparison of mucoadhesive performance and it was found as $0.324 \pm 0.036 \mathrm{~N}$. Hen's Egg Test-Chorioallantoic Membrane test showed that the formulation is nonirritant to the vaginal mucosa. Formulation F2 was subjected to accelerated stability studies at $40^{\circ} \mathrm{C} \pm 2{ }^{\circ} \mathrm{C} / 75 \% \mathrm{RH} \pm 5 \% \mathrm{RH}$ for 6 months. The results showed that it stayed steady for 6 months. Conclusion: It can be concluded that the development of a tenofovir in-situ vaginal gel which may offer effective and sustained protection against human immunodeficiency virus infection.
\end{abstract}

Key words: Antiretroviral drug, Thermosensitive, Mucoadhesive, in-situ gel, Non-irritant.

\section{INTRODUCTION}

The Human Immunodeficiency Virus (HIV) is a retrovirus, which causes a decline in the immune system and ultimately can cause Acquired Immunodeficiency Syndrome (AIDS). It is stated in the UN AIDS 2013 report that HIV continues to be driven by gender inequalities and pernicious societal practices that lead to a higher susceptibility of the female population acquiring AIDS. ${ }^{1}$ The antiretroviral therapeutic agents are preferred so as to overwhelm the HIV virus, slow down the development of HIV disease as well as avoid forward transmission of $\mathrm{HIV}^{2}$ Tenofovir, as an antiretroviral agent, is a nucleotide analog that inhibits HIV reverse transcriptase and shows potency in-vitro and in-vivo against $\mathrm{HIV}^{3}$ and so, tenofovir is one of the most common antiretroviral drug molecules used for HIV treatment. ${ }^{4,5}$ Tenofovir disoproxil fumarate, a nucleotide analog HIV-1 reverse transcriptase inhibitor is 100 times more potent in its anti-viral activity compared to its prodrug Tenofovir. ${ }^{6}$ Tenofovir disoproxil fumarate has enhanced permeability leading to a reduction in dose requirement and currently, it is administered orally in its prodrug form. Tenofovir disoproxil fumarate is effective against a range of HIV-1 subtypes, as well as CCR5-using and
Submission Date: 27-04-2020; Revision Date: 17-07-2020; Accepted Date: 23-10-2020

DOI: 10.5530/ijper.54.4.190 Correspondence:

Dr. Anita Prahladbhai Patel Assistant Professor and H.O.D., Department of Pharmaceutics, Nootan Pharmacy College, Sankalchand Patel University, Visnagar-384315, Gujarat, INDIA.

Phone no: +91-9428415211 Email id: patelanita21@gmail. com

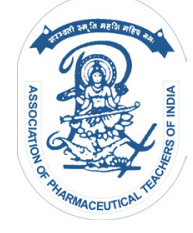

www.ijper.org 
CXCR4-using HIV-1. ${ }^{7}$ It also has a long intracellular halflife, low risk of resistance development, ${ }^{8}$ and has been proposed for use in pre-clinical trials as a microbicide. The vaginal drug delivery system was employed as a route for drug administration to achieve local plus systemic action. The benefits of the vaginal route are avoidance of the first-pass metabolism of a drug, reduction in hepatic as well as gastrointestinal side effects of the drug and overwhelming of pain, tissue damage and infection found after parenteral administration. ${ }^{10-12}$ There are two major challenges in the vaginal drug delivery system. The first one is restricted contact time triggered by the physiological conditions imposed by the protective mechanisms of the vagina and the second challenge is poor patient compliance. Conventional vaginal formulations are available in market such as vaginal ointments and inserts inducing embarrassment to the patient. ${ }^{13}$ Although it is known that patients can tolerate gels more easily as compared to vaginal ointments and inserts, but at a same time there is a difficulty in direct application of gels into the vaginal cavity. ${ }^{13}$ Mucoadhesive polymers may localize in a specific region and extend residence time, thus improve the bioavailability of drugs. ${ }^{14}$ Therefore, mucoadhesive dosage forms have become very important for the treatment of vaginal diseases. These types of dosage forms provide an interaction between the mucoadhesive polymer and vaginal mucosa and increase the residence time at the mucosal application site. Newly, the in-situ gelling systems have been found out as more opportune formulation for topical administration. The in-situ gels are stimuli-sensitive hydrogels that exist as a liquid before administration and immediately transition into gels following the contact with the vaginal mucosa. The in-situ gel offers a number of benefits for example, easy to administer into vaginal or other body cavities, excellent spreadability at certain temperatures, reduction in the frequency of administration, improvement in patient compliance and very comfortable as compared to the conventional formulations. ${ }^{15}$ The most widely employed gels are poloxamers based gels as they are easily applied and also providing good spreading as well as coating of the vagina which makes the treatment more effective and undoubtedly result in extended effect. ${ }^{16,17}$ Poloxamers were selected to develop in-situ gel which is a synthetic triblock copolymer of polyoxyethylene and polyoxypropylene that exhibits thermosensitive property in an aqueous medium. ${ }^{18}$ Poloxamers have good compatibility but has very low mucoadhesive property. So, Carbopol 934 was included in the formula for improvement in the mucoadhesive as well as mechanical properties and for confirmation of longer retention time of developed in-situ gel formulation in the vaginal cavity. The objectives of the study were to design more effective treatment for HIV by preparing a new mucoadhesive in-situ vaginal gel of Tenofovir disoproxil fumarate that adhere to the vaginal mucosa and release the drug for longer period of time to enhance the therapeutic activity of the drug. Poloxamer 407 and Carbopol 934 were employed as polymers to provide in-situ and mucoadhesive property to the system. The resulting formulation was evaluated for gelation temperature, viscosity and $\mathrm{pH}$ measurement, mucoadhesive force measurement, in-vitro release study, irritation study and stability study.

\section{MATERIALS AND METHODS}

Tenofovir disoproxil fumarate was gently supplied as gift sample by Spectrum Pharma Research Solutions, Hyderabad, Poloxamer 407 was provided by BASF, Mumbai, India as gift sample and Carbopol 934 were received from Central Drug House (P). Ltd. India. All other chemicals, as well as reagents, were of analytical reagent grade, were purchased from S.D. Fine Chemicals Ltd., Mumbai.

\section{Method of preparation of Tenofovir disoproxil fumarate vaginal in-situ gel}

Thermosensitive and mucoadhesive poloxamer based vaginal in situ forming gels of Tenofovir disoproxil fumarate was prepared by the cold technique first described by Schmolka with slight modification. ${ }^{19,20}$ Briefly, carbopol 934 was slowly added to a phosphate buffer $\mathrm{pH} 4.0$ under continuous stirring at $4^{\circ} \mathrm{C}$. Poloxamer 407 was then added gradually under continuous stirring until a clear solution was obtained. Agitation was performed in an ice bath at $4^{\circ} \mathrm{C}$. Depending on the final viscosity of the preparation and the ratio carbopol 934 and poloxamer 407, poloxamer 407 could be dissolved first and carbopol 934 at last. The mixture was then placed at temperature $4^{\circ} \mathrm{C}$ for $48 \mathrm{hr}$ to eliminate foam and air bubbles and the macroscopic homogeneity of each preparation was appreciated visually at the end of the storage period. Tenofovir disoproxil fumarate $(1 \%)$ was initially dissolved in the mixture of methanol and polyethylene glycol 400 (3:5) and added to the cold poloxamer 407 solution containing various content of carbopol 934 (0.25-1.5\%) with gentle mixing. Benzalkonium chloride $(0.01 \%)$ used as a preservative (Table 1). 


\begin{tabular}{|c|c|c|c|c|c|c|}
\hline \multirow{2}{*}{ Ingredients } & \multicolumn{6}{|c|}{ Formulation code } \\
\hline & F1 & F2 & F3 & F4 & F5 & F6 \\
\hline $\begin{array}{l}\text { Tenofovir disoproxil } \\
\text { fumarate }(\% \mathrm{w} / \mathrm{v})\end{array}$ & 1 & 1 & 1 & 1 & 1 & 1 \\
\hline $\begin{array}{l}\text { Poloxamer } 407 \text { (\% } \\
\text { w/v) }\end{array}$ & 18 & 18 & 18 & 18 & 18 & 18 \\
\hline Carbopol934 (\% w/v) & 0.25 & 0.50 & 0.75 & 1.0 & 1.2 & 1.5 \\
\hline $\begin{array}{l}\text { Benzalkonium } \\
\text { chloride ( } \% \mathrm{w} / \mathrm{v})\end{array}$ & 0.01 & 0.01 & 0.01 & 0.01 & 0.01 & 0.01 \\
\hline Water (ml) q.s. & 10 & 10 & 10 & 10 & 10 & 10 \\
\hline
\end{tabular}

\section{Drug excipients compatibility study}

A drug excipient compatibility study was performed for checking the compatibility between drug and polymers. The compatibility studies of Tenofovir disoproxil fumarate with other excipients were done by using Fourier Transform Infrared Spectroscopy (FT-IR). FT-IR spectra of pure drug and physical mixture of drug and other excipients were measured with the help of the FT-IR instrument using the Potassium Bromide $(\mathrm{KBr})$ method. The samples to be tested were mixed with solid $\mathrm{KBr}$. The mixture was then passed into a very thin pellet. The pellet was placed in the holder directly in the IR laser beam. Spectra were recorded using Shimadzu FT-IR 8400s loaded with IR solution version 1.2 software of the pure drug for any major interaction. Each spectrum was recorded in the frequency range of $3800-600 \mathrm{~cm}^{-1}$.

\section{Physicochemical characterization of Tenofovir disoproxil fumarate vaginal in-situ gel}

The clarity of the vaginal in-situ gelling formulation after and before gelling was measured by visual examination. The $\mathrm{pH}$ of the vaginal in-situ gel formulation was recorded with a digital $\mathrm{pH}$ meter and allowing equilibrating for $1 \mathrm{~min}$. The viscosity of all formulated batches of the in-situ gel was measured using Brookfield Digital Viscometer (DV-E model). The tests were performed in triplicate.

\section{Gelation temperature}

A $5 \mathrm{ml}$ aliquot of the in-situ gelling solution was transferred to a test tube, submerged in a thermostat water bath. The temperature of the water bath was raised with an increment of $1{ }^{\circ} \mathrm{C}$ and kept for 5 min to equilibrate at every new set of temperatures. After that, the sample was inspected for gelation upon slanting the test tube and it was believed to take place when the meniscus would no longer move. Each sample was measured in triplicate. ${ }^{21,22}$

\section{Drug content}

Drug content was determined by dissolving $10 \mathrm{~mL}$ Tenofovir disoproxil fumarate vaginal in situ gel in phosphate buffer $\mathrm{pH}$ 4.0. After suitable dilution absorbance was recorded by using a UV/Vis double beam Spectrophotometer at $260 \mathrm{~nm}$. The drug content was calculated with a slope of a standard curve.

\section{Mucoadhesive force determination}

The mucoadhesive force is a measure of a force necessary to separate the formulation from vaginal mucosal membrane. The apparatus urbanized for in-vitro mucoadhesive strength determination in a simulated vaginal environment is a modification of the earlier described mucoadhesion test assembly. ${ }^{23,24}$ The method is based on the measurement of shear stress required to break the adhesive bond between a model membrane and the test formulation. The Tenofovir disoproxil fumarate vaginal in-situ gelling formulation is sandwiched between two model membranes fixed supports in the assemblies for a sufficient period of time. After the formation of the adhesive bond, the weight or force needed to separate the bond will be measured and calculated as mucoadhesive strength.

\section{In-vitro drug release study}

Franz diffusion cell was used for an in-vitro drug release study with water-jacketed receptor chamber $(20 \mathrm{ml})$ and a donor chamber equipped with a thermostatically shaking water bath at $37 \pm 1^{\circ} \mathrm{C}$. The receptor chamber was containing phosphate buffer $\mathrm{pH} 4.0$ solutions was constantly stirred by a magnetic stirrer. Both the chambers separated by cellulose membrane (Filter paper Whatman

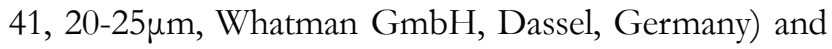
each vaginal in-situ gelling formulation spread on the circular portion of the membrane. At predetermined time intervals, $1 \mathrm{~mL}$ of the solutions were removed from the acceptor phase at each sampling time for up to $8 \mathrm{hrs}$. The aliquots were replaced with an equal volume of the freshly prepared release medium kept at the same temperature and amount of Tenofovir disoproxil fumarate release was measured by taking the absorbance at $260 \mathrm{~nm}$ against a blank with UV spectrophotometer at $260 \mathrm{~nm}$ (Shimadzu UV spectrophotometer, Japan). The result of in-vitro data was analyzed by statistical software to achieve the best fit kinetic model for in-vitro drug release from optimized formulation. The test was conducted in triplicate. ${ }^{10}$

\section{Irritation test with Hen's Egg Test Chorioallantoic Membrane Test (HET-CAM)}

For irritation study, modified HET-CAM test was performed as described by Velpandian and co-workers. ${ }^{25}$ 
In this test fertilized hen's eggs were obtained from a poultry farm. Three eggs were selected for each formulation weighing between $50-55 \mathrm{~g}$. These (special pathogen-free) eggs were incubated at $37^{\circ} \mathrm{C} \pm 0.5^{\circ} \mathrm{C}$ in a standard cell culture incubator up to day 3 . On the $3^{\text {rd }}$ day, $3 \mathrm{ml}$ of egg albumin was removed by using sterile technique through a hole made by pointed pole and then the hole sealed by $70 \%$ alcohol sterilized paraffin with the help of the heated spatula. The eggs were kept in the equatorial position for the development of CAM. The eggs were candled on the $5^{\text {th }}$ day of incubation and thereafter non-viable embryos were removed. On the $10^{\text {th }}$ day, in-situ gelling formulation was instilled directly onto CAM surface with a pipette and observed within a specific time limit. The membrane is examined for vascular damage and the time required for injury to take place is recorded.

A $0.9 \%$ Sodium Chloride $(\mathrm{NaCl})$ solution was utilized as a control as it is stated to be experimentally nonirritant. The scores were recorded according to the scoring schemes mentioned below:

Score 0 indicates no visible hemorrhage (non-irritant); Score 1 indicates just visible membrane discoloration (mild irritant); Score 2 indicates structures are covered partially due to membrane discoloration and hemorrhage (moderately irritant); Score 3 indicates structures are covered totally due to membrane discoloration and hemorrhage (severe irritant).

\section{Stability studies}

Stability studies were carried out on optimized formulation according to ICH guideline for 6 months and after that checks all the physicochemical parameters of an optimized batch of formulated Tenofovir disoproxil fumarate vaginal in-situ gel.

\section{RESULTS AND DISCUSSION \\ Drug excipients compatibility study}

The FT-IR analysis of pure drug, drug and excipients physical mixture was done on Shimadzu FT-IR 8400s. The FT-IR spectra of pure Tenofovir disoproxil fumarate and physical mixture of Tenofovir disoproxil fumarate, Carbopol 934 and poloxamer 407 are depicted in Figure 1(a) and Figure 1(b) respectively. The FT-IR spectrum of Tenofovir disoproxil fumarate showed characteristics peaks at $1645 \mathrm{~cm}^{-1}(\mathrm{C}=\mathrm{O}$ stretching), $1325 \mathrm{~cm}^{-1}\left(\mathrm{O}=\mathrm{C}=\mathrm{O}\right.$ stretching), $2910 \mathrm{~cm}^{-1}$ (Aliphatic $\mathrm{CH}$ stretching), $3000 \mathrm{~cm}^{-1}$ (Aromatic $\mathrm{CH}$ stretching), 3383 $\mathrm{cm}^{-1} \mathrm{NH}_{2}$ stretching) and $3469 \mathrm{~cm}^{-1}$ (NH stretching) were identified, which was same in the physical mixture of Tenofovir disoproxil fumarate and excipients. Thus, it was confirmed that there is no interaction between drug and excipients.

\section{Physicochemical characterization of tenofovir disoproxil fumarate vaginal in-situ gel}

The physicochemical properties such as clarity, $\mathrm{pH}$, viscosity, gelation temperature, drug content and mucoadhesive strength of the prepared formulation are depicted in Table 2.

The Tenofovir disoproxil fumarate vaginal in-situ gelling solution was transparent liquid at $4^{\circ} \mathrm{C}$ whereas transparent semisolid gel was formed at body temperature. The normal physiological $\mathrm{pH}$ of vaginal mucosa ranges from 3.5-4.5. The $\mathrm{pH}$ of all the vaginal in-situ gel formulation F1 to F6 found to be 4.0-5.0, within the range which vaginal mucosa can tolerate.

The rheological performance is a key element in the poloxamer formulation. The increase in polymer carbopol 934 concentration caused an increase in viscosity of formed vaginal in-situ gelling solution as well as vaginal in-situ gel. Results of viscosity profiles of formulations batches $\mathrm{F} 1$ to $\mathrm{F} 6$ at $37^{\circ} \mathrm{C}$ clearly indicate that the mucoadhesive polymer carbopol 934 had a viscosity-enhancing effect.

Gelation temperature is the temperature at which the liquid phase makes a transition to gel. An ideal vaginal in-situ gel should be a free-flowing liquid at room temperature for reproducible administration of formulation into the vaginal cavity where it undergoes an in-situ sol-gel phase transition to form a gel. ${ }^{26}$ The temperature of a human vagina is $37.2^{\circ} \mathrm{C},{ }^{27}$ so gelation temperature near to this temperature is a most suitable temperature. If gelation temperature is below the vaginal temperature, a gel might be formed before administration leading difficulties in manufacturing, handling and administering as well. And if gelation temperature is higher than $37.2^{\circ} \mathrm{C}$, a formulation remains in a liquid state at vaginal temperature, resulting in drainage of formulation from the vaginal cavity at an early stage.

The drug content was found to be in acceptable range (91.65- $98.58 \%$ ) for all the formulations, indicating the uniform distribution of the drug.

The mucoadhesive force is a vital and crucial characteristic for in-situ forming vaginal gels since it stops rapid drainage of formulation and therefore extends its retention time in vagina. ${ }^{28}$ The mucoadhesive strength significantly increases as the concentration of mucoadhesive polymer increased in the range of $0.25-1.5 \%$. The mucoadhesive force results indicate that the formulated Tenofovir disoproxil fumarate in-situ 
vaginal gel with poloxamer 407 had acceptable adhesive property.

\section{In-vitro drug release study}

In-vitro drug release kinetics was carried out by the use of Franz diffusion cells in order to evaluate Tenofovir disoproxil fumarate vaginal in-situ gel release profile. The release profile of Tenofovir disoproxil fumarate from all the formulations reveals that as the level of mucoadhesive polymer carbopol 943 is increasing, the drug release is decreasing due to higher viscosity of the formulation (Figure 2). The retarding effect of mucoadhesive polymer carbopol 934 could be attributed to their ability to increase the overall product viscosity as well as their ability to distort or squeeze the extra micelle aqueous channels of poloxamer

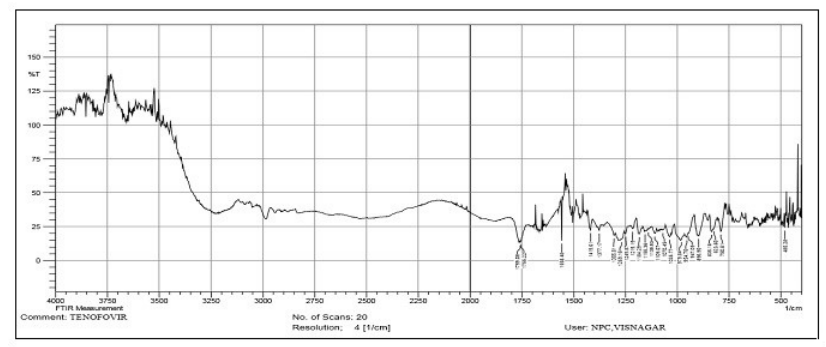

Figure 1: (a) FTIR spectrum of Tenofovir disoproxil fumarate.

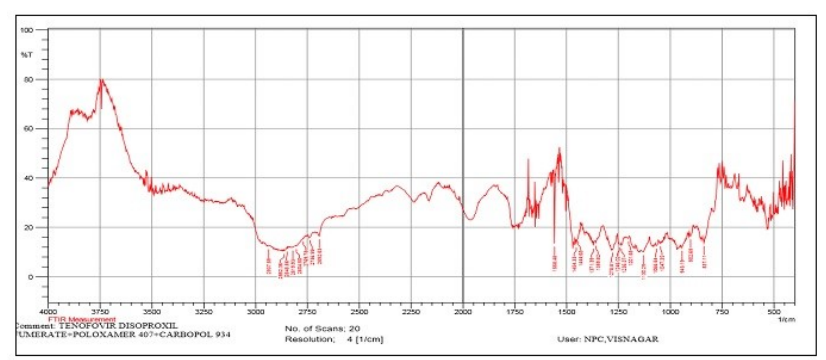

Figure 1: (b) FTIR spectrum of physical mixture of Carbopol934, Poloxamer 407 and Tenofovir disoproxil fumarate. micelle through which the drug diffuses thereby, delaying the release process. The in-vitro drug release conditions may be very different from those likely to be encountered in the vagina. However, the results clearly show that the gels have the ability to retain the drug for a prolonged period of time about $8 \mathrm{hrs}$ and that premature release will not occur.

The gels on visual inspection at periodic intervals during the in-vitro drug release experiments showed a gradual swelling after 4 hrs that resulted in an increase in the volume of most gels. No discernible relationship between the extent of swelling and gel composition could be established. Also, no apparent changes or disruptions in the integrity of the gels were noticed during the course of the experiment.

For measurement of release kinetics of Tenofovir disoproxil fumarate vaginal in-situ gel, the drug release data were analyzed by calculating coefficients of determination for evaluation of data for fitting the kinetic regression lines of zero order, first order, Higuchi, Korsmeyer-Peppas and it was observed that Korsmeyer-Peppas model showing the best correlation $\left(R^{2}=0.9860\right)$.

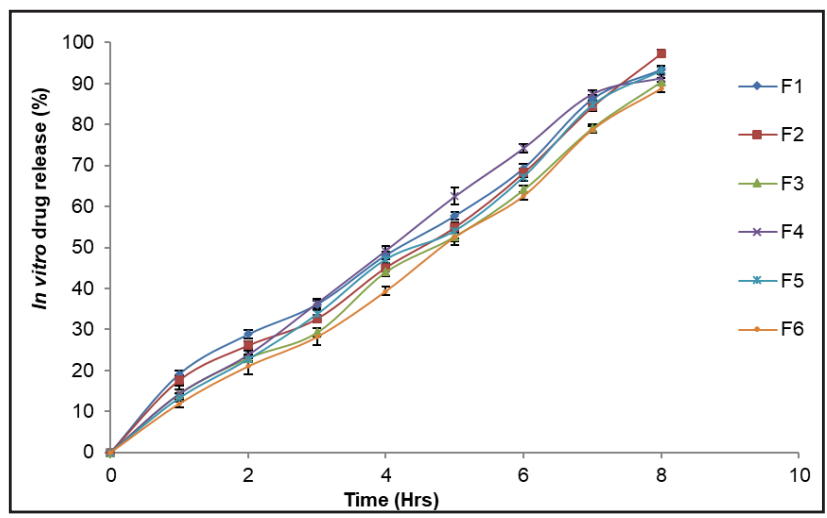

Figure 2: In-vitro drug release of formulation batches F1 to F6.

\begin{tabular}{|c|c|c|c|c|c|c|}
\hline \multicolumn{7}{|c|}{ Table 2: Physicochemical characteristics of formulation batches F1 to F6. } \\
\hline $\begin{array}{c}\text { Formulation } \\
\text { batch }\end{array}$ & pH* $^{*}$ & $\begin{array}{c}\text { Viscosity of } \text { in-situ } \\
\text { gelling solution* } \\
\text { (cps) }\end{array}$ & $\begin{array}{c}\text { Viscosity of } \\
\text { in-situ gel } \\
\text { (cps) }\end{array}$ & $\begin{array}{c}\text { Gelation } \\
\text { temperature* } \\
\text { ( }{ }^{*} \text { C) }\end{array}$ & $\begin{array}{c}\text { Drug content } \\
\text { (\%) }\end{array}$ & $\begin{array}{c}\text { Mucoadhesive } \\
\text { strength* }^{*} \\
(\mathbf{N})\end{array}$ \\
\hline F1 & $4.0 \pm 0.16$ & $160 \pm 12.25$ & $1911 \pm 0.89$ & $38 \pm 1.25$ & $95.23 \pm 0.77$ & $0.286 \pm 0.012$ \\
\hline F2 & $4.2 \pm 0.12$ & $240 \pm 8.16$ & $2116 \pm 0.62$ & $37 \pm 1.63$ & $98.58 \pm 0.89$ & $0.324 \pm 0.036$ \\
\hline F3 & $5.0 \pm 0.16$ & $360 \pm 16.33$ & $2310 \pm 0.33$ & $34 \pm 1.25$ & $96.25 \pm 0.62$ & $0.368 \pm 0.005$ \\
\hline F4 & $4.0 \pm 0.16$ & $440 \pm 12.25$ & $2580 \pm 0.60$ & $30 \pm 1.63$ & $91.65 \pm 0.79$ & $0.395 \pm 0.013$ \\
\hline F5 & $4.0 \pm 0.21$ & $520 \pm 8.16$ & $3320 \pm 0.22$ & $28 \pm 1.25$ & $92.23 \pm 0.33$ & $0.402 \pm 0.005$ \\
\hline F6 & $4.5 \pm 0.21$ & $640 \pm 16.33$ & $3532 \pm 0.89$ & $27 \pm 2.02$ & $94.98 \pm 0.62$ & $0.436 \pm 0.019$ \\
\hline
\end{tabular}

*Data are expresses as Mean \pm S.D. $(n=3)$ 
Formulation batch F2 selected as optimized formulation exhibits gelation temperature near to body temperature, acceptable $\mathrm{pH}$ for vaginal delivery, desirable mucoadhesive property to retain the drug in a vagina for a longer time, better drug content and high drug release in sustain manner up to $8 \mathrm{hr}$.

\section{Irritation test with Hen's Egg Test - Chorioallantoic Membrane Test (HET-CAM)}

Irritation of optimized formulation was tested by Hen's egg CAM test which is quick, sensitive and inexpensive. ${ }^{29}$ In addition, irritation testing of formulation with incubated eggs is a borderline case between in-vivo and in-vitro system and they do not conflict with the ethical as well as legal obligations. Irritation potential of optimized

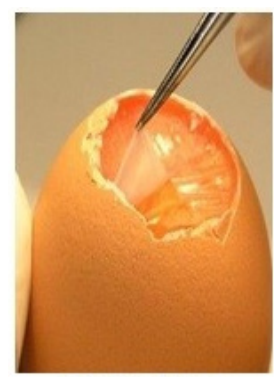

(a) Initial Phase

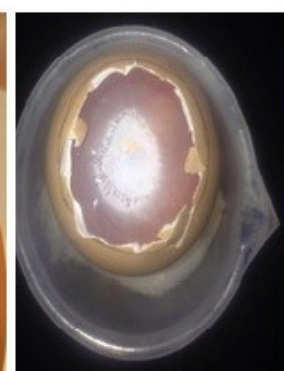

(b) After $1 \mathrm{~h}$

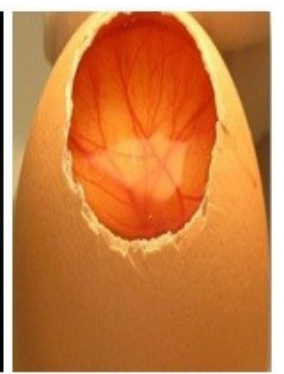

(c) After 5 hrs
Figure 3: Hen's egg CAM test for irritation study.

\section{Table 3: Score for HET-CAM test (Time in hr.).}

\begin{tabular}{|l|c|c|c|c|c|c|}
\hline Sample & $\mathbf{0}$ & $\mathbf{1}$ & $\mathbf{2}$ & $\mathbf{3}$ & $\mathbf{4}$ & $\mathbf{5}$ \\
\hline Normal saline solution as a control \\
\hline Egg 1 & 0 & 0 & 0 & 0 & 0 & 0 \\
\hline Egg 2 & 0 & 0 & 0 & 0 & 0 & 0 \\
\hline Egg 3 & 0 & 0 & 0 & 0 & 0 & 0 \\
\hline Mean & 0 & 0 & 0 & 0 & 0 & 0 \\
\hline
\end{tabular}

Optimized formulation of Tenofovir disoproxil fumarate vaginal in-situ gel

\begin{tabular}{|l|c|c|c|c|c|c|}
\hline Egg 1 & 0 & 0 & 0 & 0 & 0 & 0 \\
\hline Egg 2 & 0 & 0 & 0 & 0 & 0 & 0 \\
\hline Egg 3 & 0 & 0 & 0 & 0 & 1 & 1 \\
\hline Mean & 0 & 0 & 0 & 0 & 0.33 & 0.33 \\
\hline
\end{tabular}

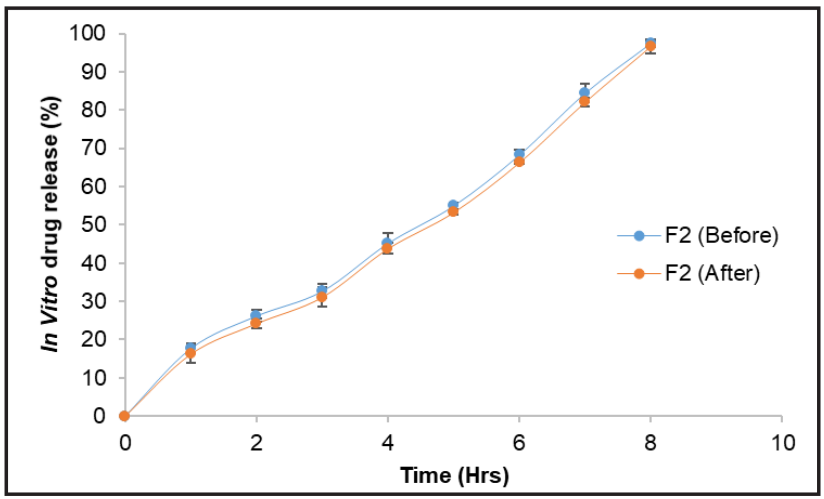

Figure 4: Release data after stability study of optimized batch (F2).

formulation was tested by using HET-CAM and results were compared with normal saline as a control that was non-irritant (Score 0) while optimized Tenofovir disoproxil fumarate vaginal in-situ gel was non-irritant up to $1 \mathrm{hr}$ (Score 0) but mean score was found to be 0.33 after $5 \mathrm{hrs}$ which indicates that formulation is nonirritant to mild-irritant (Figure 3 and Table 3).

\section{Stability studies}

A stability study was carried out on the optimized formulation of tenofovir disoproxil fumarate as per ICH guidelines for 6 months. There are no major changes are found in physicochemical characteristics as well as on release profile in optimized formulation. Results of stability studies are presented in Table 4 and Figure 4. Hence, it is indicating that during stability study the optimized formulations remain stable as per ICH guidelines. So, we can conclude that after the 6 months stability as per ICH guidelines there are no physicochemical changes are observed. Hence, our optimized formulation is stable.

\section{CONCLUSION}

The present investigation deals with the formulation of mucoadhesive Tenofovir disoproxil fumarate vaginal in-situ gel using carbopol 934 as a mucoadhesive polymer. This in-situ vaginal gel was made with the

\begin{tabular}{|c|c|c|c|c|c|}
\hline $\begin{array}{l}\text { Formulation } \\
\text { batch }\end{array}$ & $\mathrm{pH}^{*}$ & $\begin{array}{c}\text { Viscosity of in-situ } \\
\text { gel } \\
\text { (cps) }\end{array}$ & $\begin{array}{l}\text { Gelation temperature* } \\
\left({ }^{\circ} \mathrm{C}\right)\end{array}$ & $\begin{array}{c}\text { Drug content* } \\
(\%)\end{array}$ & $\begin{array}{l}\text { Mucoadhesive } \\
\text { strength* } \\
\text { (N) }\end{array}$ \\
\hline $\begin{array}{c}\text { F2 } \\
\text { (Before) }\end{array}$ & $4.2 \pm 0.12$ & $2116 \pm 0.62$ & $37 \pm 1.63$ & $98.58 \pm 0.89$ & $0.324 \pm 0.006$ \\
\hline $\begin{array}{c}\text { F2 } \\
\text { (After) }\end{array}$ & $4.0 \pm 0.08$ & $2090 \pm 0.32$ & $36 \pm 0.33$ & $98.25 \pm 0.12$ & $0.318 \pm 0.013$ \\
\hline
\end{tabular}

*Data are expressed as Mean \pm S.D. $(n=3)$ 
intention to provide a topical prophylactic dose of Tenofovir disoproxil fumarate in the vagina against HIV. The Tenofovir disoproxil fumarate vaginal in-situ gel was found to have varying degrees of mucoadhesion, retention and prolonged release based on their formula. Formulation batch F2 showed the desired mucoadhesion, retention in the vagina as well as the prolonged release of the drug up to $8 \mathrm{hrs}$. Hence, it was selected for release kinetics, where it followed the Korsmeyer-Peppas model. Formulation batch F2 was stable at the end of the accelerated stability study. Collectively, these data suggest the possibility of using the in-situ vaginal gel as a delivery system for HIV microbicides for the prevention of HIV transmission.

\section{ACKNOWLEDGEMENT}

The authors are very much grateful to Spectrum Pharma Research Solutions, Hyderabad for providing the gift sample of Tenofovir disoproxil fumarate.

\section{CONFLICT OF INTEREST}

The authors declare no conflicts of interest.

\section{ABBREVIATIONS}

HIV: Human Immunodeficiency Virus; HET-CAM: Hen's Egg Test-Chorioallantoic Membrane; AIDS: Acquired Immunodeficiency Syndrome; FT-IR: Fourier Transform Infrared Spectroscopy; UV: Ultra-Violet; KBr: Potassium Bromide; NaCl: Sodium Chloride; hrs.: Hours; Min: Minutes; q.s.: Quantity Sufficient; nm: Nanometer; ml: Milliliter; $\mu$ g: Microgram; g: Gram; cps: Centipoise; N: Newton

\section{REFERENCES}

1. Global Report: UNAIDS report on Global AIDS pandemic. Joint United Nations Programme on HIVIAIDS (UNAIDS). 2013.

2. Abdool KQ, Abdool KSS, Frohlich JA, Grobler AC, Baxter C, Mansoor LE, et al. Effectiveness and safety of tenofovir gel, an antiretroviral microbicide, for the prevention of HIV infection in women. Science. 2010;329(5996):1168-74.

3. Lee WA, He GX, Eisenberg E, Cihlar T, Swaminathan S, Mulato A, et al. Selective intracellular activation of a novel prodrug of the human immunodeficiency virus reverse transcriptase inhibitor tenofovir leads to preferential distribution and accumulation in lymphatic tissue. Antimicrob Agents Chemother. 2005;49(5):1898-906.

4. Anderson PL, Kiser JJ, Gardner EM, Rower JE, Meditz A, Grant RM. Pharmacological considerations for tenofovir and emtricitabine to prevent HIV infection. J Antimicrob Chemother. 2011;66(2):240-50.

5. Grim SA, Romanelli F. Tenofovir disoproxil fumarate. Ann Pharmacother. 2003;37(6):849-59.

6. Mesquita PMM, Rastogi R, Segarra TJ, Teller RS, Torres NM, Huber AM, et al. Intravaginal ring delivery of tenofovir disoproxil fumarate for prevention of HIV and herpes simplex virus infection. J Antimicrob Chemother. 2012;67(7):1730-8.

7. Rohan LC, Moncla BJ, Kunjara N, Ayudhya RP, Cost M, Huang Y, et al. In vitro and ex vivo testing of tenofovir shows it is effective as an HIV-1 microbicide. PLoS One. 2010;5(2):e9310.

8. Parikh UM, Dobard C, Sharma S, Cong ME, Jia H, Martin A, et al. Complete protection from repeated vaginal simian-human immunodeficiency virus exposures in macaques by a topical gel containing tenofovir alone or with emtricitabine. J Virol. 2009;83(20):10358-65.

9. Palmer S, Margot N, Gilbert H, Shaw N, JrBuckheit R, Miller M. Tenofovir, adefovir and zidovudine susceptibilities of primary human immunodeficiency virus type 1 isolates with non-B subtypes or nucleoside resistance. AIDS Res Hum Retrovir. 2001;17(12):1167-73.

10. Patel P, Patel P. Formulation and evaluation of clindamycin $\mathrm{HCl}$ in-situ gel for vaginal application. Int J Pharm Investig. 2015;5(1):50-6.

11. Neves J, Bahia MF. Gels as vaginal drug delivery systems. Int J Pharm. 2006;318(1-2):1-14.

12. Bilensoy E, Rouf MA, Vural I, Sen M, Hincal AA. Mucoadhesive, thermosensitive, prolonged-release vaginal gel for clotrimazole: $\beta$-Cyclodextrin complex. AAPS Pharm Sci Tech. 2006;7(2):E54.

13. Chang JY, Oh YK, Choi HG, Kim YB, Kim CK. Rheological evaluation of thermosensitive and mucoadhesive vaginal gels in physiological conditions. Int J Pharm. 2002a;241(1):155-63.

14. Gurny R, Meyer JM, Peppas NA. Bioadhesive intraoral release systems: Design, testing and aanalysis. Biomaterials. 1984;5(6):336-40.

15. Chang JY, Oh YK, Kong HS. Prolonged antifungal effects of clotrimazolecontaining mucoadhesive thermosensitive gels on vaginitis. J Control Release. 2002b;82(1):39-50.

16. Qui Y, Park K. Environment-sensitive hydrogels for drug delivery. Adv Drug Deliv Rev. 2001;53(3):321-39.

17. Mauck CK, Katz D, Sandefer EP, Nasution MD, Henderson M, Digenis $\mathrm{GA}$, et al. Vaginal distribution of Replens and K-Y jelly using three imaging techniques. Contraception. 2008;77(3):195-04.

18. Karavana SY, Rencber S, Senyigit Z, Baloglu E. A new in situ gel formulation of itraconazole for vaginal administration. Pharmacol Pharm. 2012;3(04):417-26.

19. Schmolka IR. Artificial skin 1, Preparation and properties of pluronic F-127 gels for treatment of burns. J Biomed Mater Res. 1972;6(6):571-82.

20. Choi HG, Jung JH, Ryu JM, Yoon SJ, Oh YK, Kim CK. Development of in situ gelling and mucoadhesive acetaminophen liquid suppository. Int J Pharm. 1998;165(1):33-44.

21. Kwon KW, Park MJ, Hwang J, Char K. Effects of alcohol addition on gelation in aqueous solution of poly (ethylene oxide)-poly (propylene oxide)-poly (ethylene oxide) triblock copolymer. Polym J. 2001;33(5):404-10.

22. Yu GE, Deng Y, Dalton S, Wang QG, Attwood D, Price C, et al. Micellization and gelation of triblock copoly (oxyethylene oxypropylene oxyethylene) F127. J Chem Soc Faraday Trans. 1992;88(17):2537-44.

23. Spielmann H. Ocular irritation. In: Castle JV, Gomez MJ, editors. In vitro Methods in Pharmaceutical Research. $2^{\text {nd }}$ ed: Academic Press. 1997;265-87.

24. Gupta A, Garg S, Khar RK. Measurement of Bioadhesive Strength of Mucoadhesive Buccal Tablets: Design of an in vitro Assembly. Indian Drugs. 1993;30(4):152-5.

25. Velpandian T, Bankoti R, Humayun S, Ravi AK, Kumari SS, Biswas NR. Comparative evaluation of possible ocular photochemical toxicity of fluoroquinolones meant for ocular use in experimental models. Indian J Exp Biol. 2006;44(5):387-91.

26. Hussain A, Ahsan F. The vagina as a route for systemic drug delivery. $J$ Control Release. 2005;103(2):301-13.

27. Kim EY, Gao ZG, Park JS, Han K. RhEGF/HP-beta-CD complex in poloxamer gel for ophthalmic delivery. Int J Pharm. 2002;233(1-2):159-67.

28. Patel A, Patel J. Sertaconazole in-situ gel for vulvovaginal candidiasis. The Pharma Review. 2019:115-8.

29. Mortazavi SA, Carpenter BG, Smart JD. An investigation of the rheological behavior of the mucoadhesive/mucosal interface. Int J Pharm. 1992;83(13):221-5. 


\section{PICTORIAL ABSTRACT}

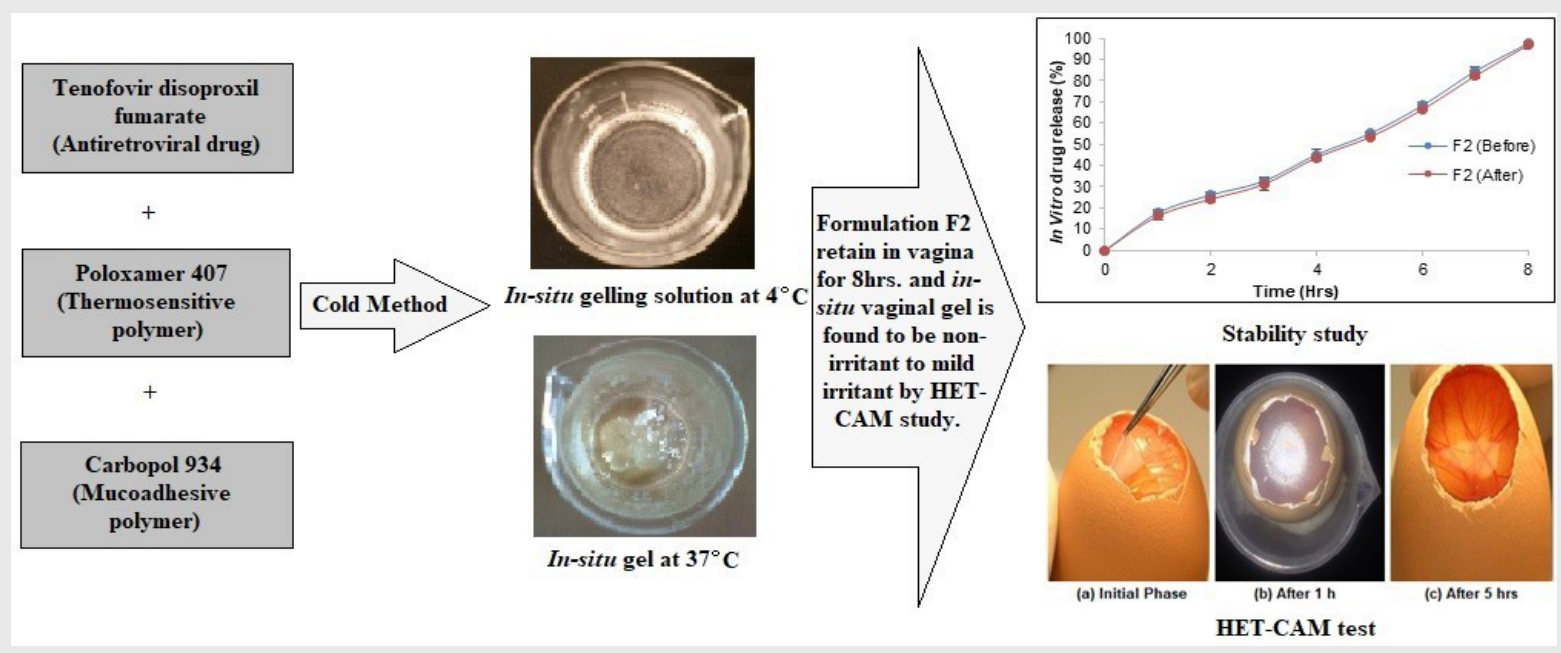

\section{SUMMARY}

Tenofovir disoproxil fumarate is selected as a drug because it is one of the most common antiretroviral drug molecules used for HIV treatment. The objectives of the present investigation was to fabricate thermosensitive mucoadhesive in-situ vaginal gel of Tenofovir disoproxil fumarate to provide topical prophylactic dose of drug in vagina for HIV infection and also bring about extended retention of dosage from in the vaginal cavity. The vaginal in-situ gel of Tenofovir disoproxil fumarate was formulated using thermosensitive polymer poloxamer 407 and mucoadhesive polymer carbopol 934 by cold method. Prepared in-situ gel were characterized for drug-excipient compatibility, physicochemical characteristics like viscosity, $\mathrm{pH}$ and appearance, gelation temperature, in-vitro drug release study, mucoadhesive study, stability study and irritation study by HET-CAM Test. Drug excipient compatibility study revealed that there is no interaction between drug and excipients by means of FT-IR study. On the basis of evaluation parameters, Formulation F2 was selected as most appropriate formulation because it displayed desired mucoadhesion, retention in the vagina as well as the prolonged release of drug up to $8 \mathrm{hr}$. exploit it in therapy of HIV. It was also exhibited good mucoadhesive force $(0.324 \pm 0.036 \mathrm{~N})$ to retain formulation in vaginal cavity for longer period of time. Irritation study results revealed that the formulation is non-irritant to the vaginal mucosa. Stability study results showed that it was stayed steady for 6 months. Finally, these data suggesting the possibility of using the in-situ vaginal gel as a delivery system for sustained protection against HIV infection.

\section{About Authors}

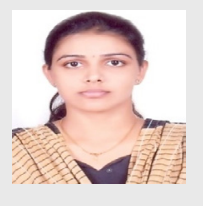

Dr. Anita Patel, Assistant Professor and H.O.D., Department of Pharmaceutics, Nootan Pharmacy College, Sankalchand Patel University, Visnagar-384315, Gujarat, India.

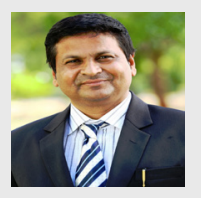

Dr. Jayvadan K. Patel, Dean and Principal, Department of Pharmaceutics, Faculty of Pharmacy, Nootan Pharmacy College, Sankalchand Patel University, Visnagar - 384315, Gujarat, India.

Cite this article: Patel AP, Patel JK. Mucoadhesive in-situ Gel Formulation for Vaginal Delivery of Tenofovir Disoproxil Fumarate. Indian J of Pharmaceutical Education and Research. 2020;54(4):963-70. 